\title{
Optimal control problem for stationary quasi-optic equations
}

Yusuf Koçak ${ }^{1 *}$ and Ercan Çelik²

${ }^{\text {"Correspondence: }}$ ykocak27@hotmail.com

${ }^{1}$ Department of Mathematics, Ağrı İbrahim Çeçen University Faculty of Science and Art, Ağrı, Turkey Full list of author information is available at the end of the article

\begin{abstract}
In this paper, an optimal control problem was taken up for a stationary equation of quasi optic. For the stationary equation of quasi optic, at first judgment relating to the existence and uniqueness of a boundary value problem was given. By using this judgment, the existence and uniqueness of the optimal control problem solutions were proved. Then we state a necessary condition to an optimal solution. We proved differentiability of a functional and obtained a formula for its gradient. By using this formula, the necessary condition for solvability of the problem is stated as the variational principle.
\end{abstract}

Keywords: stationary equation of quasi optic; boundary value problem; optimal control problem; variational problem

\section{Introduction}

Optimal control theory for the quantum mechanic systems described with the Schrödinger equation is one of the important areas of modern optimal control theory. Actually, a stationary quasi-optics equation is a form of the Schrödinger equation with complex potential. Such problems were investigated in [1-5]. Optimal control problem for nonstationary Schrödinger equation of quasi optics was investigated for the first time in [6].

\section{Formulation of the problem}

We are interested in finding the problem of the minimum of the functional

$$
J_{\alpha}(v)=\|\psi(\cdot, L)-y\|_{L_{2}(0, l)}^{2}+\alpha\|v-\omega\|_{H}^{2}
$$

in the set

$$
\begin{aligned}
V \equiv & \left\{v=\left(v_{0}, v_{1}, \varphi_{0}, \varphi_{1}\right), v_{m} \in L_{2}(0, l), v_{1}(z) \geq 0, \stackrel{0}{\forall z \in(0, L),}\right. \\
& \left.\left\|v_{m}\right\|_{L_{2}(0, l)} \leq b_{m}, \varphi_{m} \in L_{2}(0, l),\left\|\varphi_{m}\right\|_{L_{2}(0, l)} \leq d_{m}, m=0,1\right\}
\end{aligned}
$$

under the condition

$$
\begin{aligned}
& i \frac{\partial \psi}{\partial z}+a_{0} \frac{\partial^{2} \psi}{\partial x^{2}}+v_{0}(z) \psi+i \nu_{1}(z) \psi=f(x, z), \quad(x, z) \in \Omega, \\
& \psi(x, 0)=\varphi(x)=\varphi_{0}(x)+i \varphi_{1}(x), \quad x \in(0, l), \\
& \psi(0, z)=\psi(l, z)=0, \quad z \in(0, L),
\end{aligned}
$$

๑ 2012 Koçak and Çelik; licensee Springer. This is an Open Access article distributed under the terms of the Creative Commons Attribution License (http://creativecommons.org/licenses/by/2.0), which permits unrestricted use, distribution, and reproduction in any medium, provided the original work is properly cited. 
where $i=\sqrt{-1}, a_{0}>0, l>0, L>0, \alpha \geq 0, b_{0} \geq 0, b_{1}>0, d_{0}>0, d_{1}>0$ are numbers, $x \in[0, l], z \in[0, L], \Omega_{z}=(0, l) \times(0, z), \Omega=\Omega_{\mathrm{L}}, y(x), f(x, z)$ are complex valued measurable functions and satisfy the conditions

$$
\begin{aligned}
& f \in L_{2}(\Omega), \\
& y \in L_{2}(0, l)
\end{aligned}
$$

respectively, $\omega=\left(\omega_{0}, \omega_{1}, \varpi_{0}, \varpi_{1}\right)$ and $H=\left(L_{2}(0, l)\right)^{2} \times\left(L_{2}(0, L)\right)^{2} . L_{2}(0, l)$ is a Hilbert space that consists of all functions in $(0, l)$, which are measurable and square-integrable. $L_{2}(\Omega)$ is the well-known Lebesgue space consisting of all functions in $\Omega$, which are measurable and square-integrable.

The problem of finding a function $\psi=\psi(x, \mathrm{z}) \equiv \psi(x, z ; v)$ under the condition (2)-(4) for each $\forall v \in V$, which is a boundary value problem, is a function for Eq. (2).

Generalized solution of this problem is a function $\psi=\psi(x, \mathrm{z}) \equiv \psi(x, z ; v)$ belonging to the $C^{0}\left([0, L], L_{2}(0, l)\right)$, and it satisfies the integral identity

$$
\begin{aligned}
& \int_{\Omega} \psi\left(i \frac{\partial \bar{\eta}}{\partial z}+a_{0} \frac{\partial^{2} \bar{\eta}}{\partial x^{2}}+v_{0}(z) \eta+i v_{1}(z) \eta\right) d x d z \\
& \quad=\int_{\Omega} f \bar{\eta} d x d z-i \int_{0}^{l} \psi(x, L) \bar{\eta}(x, L) d x+\int_{0}^{l}\left(\varphi_{0}(x)+i \varphi_{1}(x)\right) \bar{\eta}(x, 0) d x
\end{aligned}
$$

for $\forall \eta \in C^{0}\left([0, L], L_{2}(0, l)\right)$.

\section{Existence and uniqueness of a solution of the optimal control problem}

In this section, we prove the optimal control problem using the Galerkin method and the existence and uniqueness of a solution of the problem (1)-(4).

Theorem 1 Suppose that a function $f$ satisfies the condition (5). So, for each $\forall v \in V$, the problem (2)-(4) has a unique solution, and for this solution, the estimate

$$
\|\psi(\cdot, z)\|_{L_{2}(0, l)}^{2} \leq c_{0}\left(\|\varphi\|_{L_{2}(0, l)}^{2}+\|f\|_{L_{2}(\Omega)}^{2}\right)
$$

is valid for $\forall z \in[0, L]$. Here, the number $c_{0}>0$ is independent of $z$.

Proof Proof can be done by processes similar to those given in [7].

Theorem 2 Let us accept that the conditions of Theorem 1 hold and $y \in L_{2}(0, l)$ is a given function. Then there is such a set $G$ dense in $H \equiv\left[L_{2}(0, L)\right]^{2} \times\left[L_{2}(0, l)\right]^{2}$ that the optimal control problem (1)-(4) has a unique solution $\forall \omega \in G$ and $\alpha>0$.

Proof Firstly, let us show that

$$
J_{0}(v)=\|\psi(\cdot, L)-y\|_{L_{2}(0, l)}^{2}
$$

is continuous on the set $V$. Let us take an arbitrary $\in V$, and let $v+\Delta v$ be an increment of the $v$ for the $\Delta v \in H$. Then the solution $\psi(x, z ; v)$ of the problem (1)-(4) will have an increment $\Delta \psi=\Delta \psi(x, z)=\psi(x, z ; v+\Delta v)-\psi(x, z ; v)$. Here, the function $\psi_{\Delta}(x, z)=$ $\psi(x, z ; v+\Delta v)$ is the solution of (2)-(4). On the basis of the assumptions and conditions 
(2)-(4), it can be shown that the function $\Delta \psi(x, z)$ is a solution of the following boundary value problem:

$$
\begin{aligned}
& i \frac{\partial \Delta \psi}{\partial z}+a_{0} \frac{\partial^{2} \Delta \psi}{\partial x^{2}}+\left(v_{0}(z)+\Delta v_{0}(z)\right) \Delta \psi+i\left(v_{1}(z)+\Delta v_{1}(z)\right) \Delta \psi \\
& \quad=-\Delta v_{0}(z) \psi-i v_{1}(z) \psi, \quad(x, z) \in \Omega, \\
& \Delta \psi(x, 0)=\Delta \varphi_{0}(x)+i \Delta \varphi_{1}(x), \quad x \in(0, l), \\
& \Delta \psi(0, z)=\Delta \psi(l, z)=0, \quad z \in(0, L) .
\end{aligned}
$$

Because the problem (10)-(12) and the problem (2)-(4) are the same type problems, we can write the following estimate the same as (8):

$$
\|\Delta \psi(\cdot, z)\|^{2} \leq c_{4}\|\Delta \psi\|_{L_{2}(0, l)}^{2}+\left\|\Delta v_{0} \psi+i \Delta v_{1} \psi\right\|_{L_{2}(\Omega)}^{2}, \quad \forall z \in[0, L]
$$

If we use estimate (13) then we can write the following estimate:

$$
\|\Delta \psi(\cdot, z)\|_{L_{2}(0, l)}^{2} \leq c_{5}\|\Delta v\|_{H}^{2}, \quad \forall z \in[0, L]
$$

$c_{5}>0$ is constant that does not depend on $\Delta v$.

Now, let us evaluate the increment of the functional $J_{0}(v)$ on $v \in V$. Using formula (9) we can write the equality as

$$
\begin{aligned}
\Delta J_{0}(v) & =J_{0}(v+\Delta v)-J_{0}(v) \\
& =2 \int_{0}^{l} \operatorname{Re}(\psi(x, L)-y(x)) \Delta \bar{\psi}(x, L) d x+\|\Delta \psi(\cdot, L)\|_{L_{2}(0, l)}^{2} .
\end{aligned}
$$

Using the Cauchy-Bunyakowski inequality and estimates (8) and (14), we write the inequality as

$$
\left|\Delta J_{0}(v)\right| \leq c_{6}\|\Delta v\|_{H}^{2}, \quad \forall v \in V
$$

where $c_{6}>0$ is a constant that does not depend on $\Delta v$. This inequality shows that the functional $J_{0}(v)$ is continuous on the set $V$. On the other hand, $J_{0}(z) \geq 0$ for $\forall z \in V$; therefore, $J_{0}(v)$ is bounded on $V$. The set $V$ is closed, bounded on a Hilbert space $H$. According to Theorem (Goebel) in [8], there is such a set $G$ dense in $H$ that optimalcontrol problem (1)-(4) has a unique solution for $\alpha>0$ and $\forall \omega \in G$. Theorem 2 is proven.

\subsection{Fréchet diffrentiability of the functional}

In this section, we prove the Fréchet differentiability of a given functional. For this purpose, we consider the following adjoint boundary value problem:

$$
\begin{aligned}
& i \frac{\partial \varphi}{\partial z}+a_{0} \frac{\partial^{2} \varphi}{\partial x^{2}}+v_{0}(z) \varphi-i v_{1}(z) \varphi=0, \quad(x, z) \in \Omega, \\
& \varphi(x, L)=-2 i(\psi(x, L)-y(x)), \quad x \in(0, l), \\
& \varphi(0, z)=\varphi(l, z)=0, \quad z \in(0, L) .
\end{aligned}
$$


Here, the function $\psi=\psi(x, z) \equiv \psi(x, z ; v)$ is a solution of (2)-(4) for $v \in V$. The solution of the boundary value problem (17)-(19) corresponding to $v \in V$ is a function $\varphi=\varphi(x, z)$ that belongs to the space $C^{0}\left([0, L], L_{2}(0, L)\right)$ and satisfies the integral identity

$$
\begin{aligned}
& \int_{\Omega} \varphi\left(-i \frac{\partial \bar{\eta}_{1}}{\partial z}+a_{0} \frac{\partial^{2} \bar{\eta}_{1}}{\partial x^{2}}+v_{0}(z) \bar{\eta}_{1}-i v_{1}(z) \bar{\eta}_{1}\right) d x d z \\
& =-2 \int_{0}^{l}(\psi(x, L)-y(x)) \bar{\eta}_{1}(x, L) d x+i \int_{0}^{l} \varphi(x, 0) \bar{\eta}_{1}(x, 0) d x \\
& \quad \text { for } \forall \eta_{1} \in \stackrel{0}{W}_{2}^{2,1}(\Omega) .
\end{aligned}
$$

As seen, the problem (17)-(19) is an initial boundary value problem. This can easily be obtained by a transform $\theta=L-z$. Actually, if we do a variable transform $\theta=L-z$, we obtain the boundary problem as

$$
\begin{aligned}
& i \frac{\partial \tilde{\varphi}}{\partial \theta}+a_{0} \frac{\partial^{2} \tilde{\varphi}}{\partial x^{2}}+\tilde{v}_{0}(\theta) \tilde{\varphi}-i \tilde{v}_{1}(\theta) \tilde{\varphi}=0, \quad \forall(x, \theta) \in \Omega, \\
& \tilde{\varphi}(x, 0)=-2 i(\psi(x, L)-y(x)), \quad x \in(0, l) \\
& \tilde{\varphi}(0, \theta)=\tilde{\varphi}(l, \theta)=0, \quad z \in(0, L),
\end{aligned}
$$

where

$$
\begin{aligned}
& \tilde{\varphi}(x, v)=\varphi(x, L-T)=\varphi(x, z), \quad \tilde{v}_{0}(\theta)=v_{0}(L-\theta)=v_{0}(z) \\
& \tilde{v}_{0}(\theta)=v_{0}(L-\theta)=v_{0}(z) .
\end{aligned}
$$

If we write the complex conjugate of this boundary value problem, we obtain the following boundary value problem:

$$
\begin{aligned}
& i \frac{\partial F}{\partial \theta}+a_{0} \frac{\partial^{2} F}{\partial x^{2}}+\tilde{v}_{0}(\theta) F-i \tilde{v}_{1}(\theta) F=0, \quad \forall(x, z) \in \Omega, \\
& F(x, 0)=h(x), \quad x \in(0, l) \\
& F(0, v)=F(l, \theta)=0, \quad \theta \in(0, L),
\end{aligned}
$$

where

$$
F(x, \theta)=\overline{\tilde{\varphi}(x, \theta)}, \quad h(x)=-2 i(\bar{\psi}(x, L)-\bar{y}(x))
$$

This problem is a type of (2)-(4) boundary value problem. As the right-hand side is equal to zero, and initial function $h(x)$ belongs to the space $L_{2}(0, l)$ for $\psi \in C^{0}\left([0, L], L_{2}(0, l)\right)$, $y \in L_{2}(0, l)$. By using Theorem 2, it follows that the solution of the bounded value problem (24)-(26) existing in the space $C^{0}\left([0, L], L_{2}(0, l)\right)$ is unique, and the following estimate is obtained:

$$
\|F(\cdot, \theta)\|_{L_{2}(0, l)}^{2} \leq c_{7}\|h\|_{L_{2}(0, l)}^{2}, \quad \forall \theta \in[0, L]
$$

If we use the problem (24)-(26) as a type of the conjugate problem (17)-(19), we obtain the initial bounded value problem (17)-(19) has a unique solution belonging to the space 
$C^{0}\left([0, L], L_{2}(0, l)\right)$, and the following estimate is obtained:

$$
\|\varphi(\cdot, z)\|_{L_{2}(0, l)}^{2} \leq c_{8}\|\psi(\cdot, L)-y\|_{L_{2}(0, l)}^{2}, \quad \forall z \in[0, L] .
$$

Here, the number $c_{8}>0$ is independent of $\psi$ and $y$. Now, using estimate (8) in this inequality, we easily write the following estimate:

$$
\|\varphi(\cdot, z)\|_{L_{2}(0, l)}^{2} \leq c_{9}\left(\|\varphi\|_{L_{2}(0, l)}^{2}+\|y\|_{L_{2}(0, l)}^{2}+\|\varphi\|_{L_{2}(\Omega)}^{2}\right), \quad \forall z \in[0, L]
$$

Here, the number $c_{9}>0$ is constant.

Theorem 3 Let us accept that the conditions of Theorem 2 hold and $\omega \in H$ is given. Then the functional $J_{\alpha}(v)$ can be Frechet differentiable in the set $V$ and the formula below for a gradient of the functional is valid:

$$
\begin{gathered}
J_{\alpha}^{\prime}(v)=\left(J_{\alpha v_{0}}^{\prime}(v), J_{\alpha v_{1}}^{\prime}(v), J_{\alpha \varphi_{0}}^{\prime}(v), J_{\alpha \varphi_{1}}^{\prime}(v)\right), \text { where } \\
J_{\alpha \nu_{0}}^{\prime}(v)=\int_{0}^{l} \operatorname{Re}(\psi \bar{\varphi}) d x+2 \alpha\left(v_{0}(z)-\omega_{0}(z)\right), \\
J_{\alpha \nu_{1}}^{\prime}(v)=-\int_{0}^{l} \operatorname{Im}(\psi \bar{\varphi}) d x+2 \alpha\left(v_{1}(z)-\omega_{1}(z)\right), \\
J_{\alpha \varphi_{0}}^{\prime}(v)=\operatorname{Im}(\bar{\varphi}(x, 0))+2 \alpha\left(\varphi_{0}(x)-\tilde{\omega}_{0}(x)\right), \\
J_{\alpha \varphi_{1}}^{\prime}(v)=\operatorname{Re}(\bar{\varphi}(x, 0))+2 \alpha\left(\varphi_{1}(x)-\tilde{\omega}_{1}(x)\right) .
\end{gathered}
$$

Proof Let us evaluate the increment of the functional $J_{\alpha}(v)$ for the element $\forall v \in V$. We can write the following equation for the increment of the functional:

$$
\begin{aligned}
\Delta J_{\alpha}(v)= & J_{\alpha}(v+\Delta v)-J_{\alpha}(v) \\
= & 2 \int_{0}^{l} \operatorname{Re}[(\psi(x, L)-y(x)) \Delta \bar{\psi}(x, L)] d x+2 \alpha \int_{0}^{l}\left(\varphi_{0}(x)-\tilde{\omega}_{0}(x)\right) \Delta \varphi_{0}(x) d x \\
& +2 \alpha \int_{0}^{l}\left(\varphi_{1}(x)-\tilde{\omega}_{1}(x)\right) \Delta \varphi_{1}(x) d x+2 \alpha \int_{0}^{T}\left(v_{0}(z)-\omega_{0}(z)\right) \Delta v_{0}(z) d z \\
& +2 \alpha \int_{0}^{T}\left(v_{1}(z)-\omega_{1}(z)\right) \Delta v_{1}(z) d z+\|\Delta \psi(\cdot, L)\|_{L_{2}(0, l)}^{2}+\alpha\|\Delta v\|_{H^{*}}^{2} .
\end{aligned}
$$

The last formula can be written as follows:

$$
\begin{aligned}
\Delta J_{\alpha}(v)= & J_{\alpha}(v+\Delta v)-J_{\alpha}(v) \\
= & \int_{0}^{L}\left(\int_{0}^{l} \operatorname{Re}(\psi \bar{\varphi}) d x+2 \alpha\left(v_{0}(z)-\omega_{0}(z)\right) \Delta v_{0}(z) d z-\int_{0}^{l} \operatorname{Im}(\psi, \bar{\varphi}) d x \Delta v_{1}(z)\right) \\
& +\int_{0}^{L}\left(-\int_{0}^{l} \operatorname{Im}(\psi \bar{\varphi}) d x+2 \alpha\left(v_{1}(z)-\omega_{1}(z)\right)\right) \Delta v_{1}(z) d z \\
& +\int_{0}^{l}\left[\operatorname{Im}(\bar{\varphi}(x, 0))+2 \alpha\left(\varphi_{0}(x)-\tilde{\omega}_{0}(x)\right)\right] \Delta \varphi_{0}(x) d x \\
& +\int_{0}^{l}\left[\operatorname{Re}(\bar{\varphi}(x, 0))+2 \alpha\left(\varphi_{1}(x)-\tilde{\omega}_{1}(x)\right)\right] \Delta \varphi_{1}(x) d x+R(\Delta v),
\end{aligned}
$$


where $R(\Delta v)$ is defined as the formula

$$
\begin{aligned}
R(\Delta v)= & \|\Delta \psi(\cdot, L)\|_{L_{2}(0, l)}^{2}+\alpha\|\Delta v\|_{H}^{2} \\
& +\int_{\Omega} \operatorname{Re}(\Delta \psi \bar{\varphi}) \Delta v_{0}(z) d x d z \\
& -\int_{\Omega} \operatorname{Im}(\Delta \psi \bar{\varphi}) \Delta v_{1}(z) d x d z .
\end{aligned}
$$

Applying the Cauchy-Bunyakowski inequality, we obtain:

$$
\begin{aligned}
|R(\Delta v)| \leq & \|\Delta \psi(\cdot, L)\|_{L_{2}(0, l)}^{2}+\alpha\|\Delta v\|_{H}^{2} \\
& +\left(\left\|\Delta v_{1}\right\|_{L_{2}(0, T)}+\left\|\Delta v_{0}\right\|_{L_{2}(0, T)}\right) \max \|\Delta \psi(\cdot, L)\|_{L_{2}(0, l)}\|\varphi\|_{L_{2}(0, L)} .
\end{aligned}
$$

If we use estimates (13) and (28) in this inequality, we obtain

$$
|R(\Delta v)| \leq c_{10}\|\Delta v\|_{H}^{2}
$$

Here, $c_{10}>0$ is a constant that does not depend on $\Delta v$. Hence, we write

$$
R(\Delta v)=o\left(\|\Delta v\|_{H}\right)
$$

By using equality (33), the increment of the functional can be written as

$$
\begin{aligned}
& \int_{0}^{T}\left(\int_{0}^{l} \operatorname{Re}(\psi \bar{\varphi}) d x+2 \alpha\left(v_{0}(z)-\omega_{0}(z)\right) \Delta v_{0}(z) d z-\int_{0}^{l} \operatorname{Im}(\psi, \bar{\varphi}) d x \Delta v_{1}(z)\right) \\
& \quad+\int_{0}^{T}\left(-\int_{0}^{l} \operatorname{Im}(\psi \bar{\varphi}) d x+2 \alpha\left(v_{1}(z)-\omega_{1}(z)\right) \Delta v_{1}(z) d z\right. \\
& \quad+\int_{0}^{l}\left[\operatorname{Im}(\bar{\varphi}(x, 0))+2 \alpha\left(\varphi_{0}(x)-\tilde{\omega}_{0}(x)\right)\right] \Delta \varphi_{0}(x) d x \\
& \quad+\int_{0}^{l}\left[\operatorname{Re}(\bar{\varphi}(x, 0))+2 \alpha\left(\varphi_{1}(x)-\tilde{\omega}_{1}(x)\right)\right] \Delta \varphi_{1}(x) d x+o\left(\|\Delta v\|_{H}\right) .
\end{aligned}
$$

Considering this equality (34), and by using the definition of Fréchet differentiable, we can easily obtain the validity of the rule. Theorem 3 is proved.

\subsection{A necessary condition for an optimal solution}

In this section, we prove the continuity of a gradient and state a necessary condition to an optimal solution in the variational inequality form using the gradient.

Theorem 4 Accept that the conditions of Theorem 3 hold and $v^{*} \in V$ is an optimal solution of the problem (1)-(4). Then the following inequality is valid for $\forall v \in V$ :

$$
\begin{aligned}
& \int_{0}^{L}\left[\int_{0}^{l} \operatorname{Re}\left(\psi^{*}(x, z) \bar{\varphi}^{*}(x, z) d x+2 \alpha\left(v_{0}^{*}(z)-\omega_{0}(z)\right)\right)\right]\left(v_{0}(z)-v_{0}^{*}(z)\right) d z \\
& \quad+\int_{0}^{L}\left[-\int_{0}^{l} \operatorname{Im}\left(\psi^{*}(x, z) \bar{\varphi}^{* *}(x, z) d x+2 \alpha\left(v_{1}^{*}(z)-\omega_{1}(z)\right)\right)\right]\left(v_{1}(z)-v_{1}^{*}(z)\right) d z
\end{aligned}
$$




$$
\begin{aligned}
& +\int_{0}^{l}\left[\operatorname{Im}\left(\bar{\varphi}^{*}(x, 0)\right)+2 \alpha\left(\varphi_{0}^{*}(x)-\tilde{\omega}_{0}(x)\right)\right]\left(\varphi_{0}(x)-\varphi_{0}^{*}(z)\right) d x \\
& +\int_{0}^{l}\left[\operatorname{Re}\left(\bar{\varphi}^{*}(x, 0)\right)+2 \alpha\left(\varphi_{1}^{*}(x)-\tilde{\omega}_{1}(x)\right)\right]\left(\varphi_{1}(x)-\varphi_{1}^{*}(z)\right) d x \geq 0 .
\end{aligned}
$$

Here, the functions $\psi^{*}(x, z) \equiv \psi\left(x, z ; v^{*}\right), \varphi^{*}(x, z) \equiv \varphi\left(x, z ; v^{*}\right)$ are solutions of the problems (2)-(4) and a conjugate problem corresponding to $v^{*} \in V$, respectively.

Proof Now, we prove that the gradient $J_{\alpha}^{\prime}(v)$ is continuous at $V$. For this we show

$$
\begin{aligned}
& \left\|J_{\alpha v_{0}}^{\prime}(v+\Delta v)-J_{\alpha v_{0}}(v)\right\|_{L_{2}(0, L)} \rightarrow 0, \\
& \left\|J_{\alpha v_{1}}^{\prime}(v+\Delta v)-J_{\alpha v_{1}}(v)\right\|_{L_{2}(0, L)} \rightarrow 0, \\
& \left\|J_{\alpha \varphi_{0}}^{\prime}(v+\Delta v)-J_{\alpha \varphi_{0}}(v)\right\|_{L_{2}(0, l)} \rightarrow 0, \\
& \left\|J_{\alpha \varphi_{1}}^{\prime}(v+\Delta v)-J_{\alpha \varphi_{1}}(v)\right\|_{L_{2}(0, l)} \rightarrow 0
\end{aligned}
$$

for $\|\Delta v\|_{H} \rightarrow 0$.

In order to show (36), using the formula $J_{\alpha v_{0}}^{\prime}(v)=\int_{0}^{l} \operatorname{Re}(\psi \bar{\varphi}) d x+2 \alpha\left(v_{0}(z)-\omega_{0}(z)\right)$ in (29), we can write the following equation:

$$
\begin{aligned}
J_{\alpha \nu_{0}}^{\prime}(v+\Delta v)-J_{\alpha \nu_{0}}(v) \\
=\int_{0}^{l} \operatorname{Re}\left(\psi_{\Delta} \bar{\varphi}_{\Delta}\right) d x+2 \alpha\left(v_{0}(z)+\Delta v_{0}(z)-\omega_{0}(z)\right) \\
\quad \quad-\int_{0}^{l} \operatorname{Re}(\psi \bar{\varphi}) d x+2 \alpha\left(v_{0}(z)-\omega_{0}(z)\right) \\
=\int_{0}^{l} \operatorname{Re}\left(\psi_{\Delta} \bar{\varphi}_{\Delta}-\psi \bar{\varphi}\right) d x+2 \alpha \\
=\int_{0}^{l} \operatorname{Re}(\psi(x, z ; v+\Delta v) \bar{\varphi}(x, z ; v+\Delta v)-\psi(x, z ; v) \bar{\varphi}(x, z ; v)) d x+2 \alpha \Delta v(z) \\
=\int_{0}^{l} \operatorname{Re}\left(\psi_{\Delta}(x, z) \Delta \bar{\varphi}(x, z)-\Delta \psi(x, z) \bar{\varphi}(x, z)\right) d x+2 \alpha \Delta v(z) .
\end{aligned}
$$

Here, $\Delta \psi=\Delta \psi(x, z)$ is the solution of the problem (9)-(11) and $\Delta \varphi=\Delta \varphi(x, z)$ is the solution of the following problem:

$$
\begin{aligned}
& i \frac{\partial \Delta \varphi}{\partial z}+a_{0} \frac{\partial^{2} \Delta \varphi}{\partial x^{2}}+\left(v_{0}(z)-\Delta v_{0}(z)\right) \Delta \varphi-i\left(v_{1}(z)-\Delta v_{1}(z)\right) \Delta \varphi \\
& \quad=-\Delta v_{0}(z) \varphi+i \Delta v_{1}(z) \varphi, \quad(x, z) \in \Omega, \\
& \Delta \varphi(x, L)=-2 i \Delta \psi(x, L), \quad x \in(0, l), \\
& \Delta \varphi(0, z)=\Delta \varphi(l, z)=0, \quad z \in(0, L) .
\end{aligned}
$$

This bounded value problem is a type of a conjugate problem. For this solution, the following estimate is valid:

$$
\|\varphi(\cdot, z)\|_{L_{2}(0, l)}^{2} \leq c_{11}\left(\left\|\Delta v_{0} \varphi+i \Delta v_{0} \varphi\right\|_{L_{2}(\Omega)}^{2}+\|\Delta \psi(\cdot, L)\|_{L_{2}(0, l)}^{2}\right), \quad \forall z \in(0, L) .
$$


Here, the number $c_{11}$ is constant.

Using (13) and (28), we write

$$
\|\varphi(\cdot, z)\|_{L_{2}(0, l)}^{2} \leq c_{12}\left(\|\Delta v\|_{H}^{2}\right), \quad \forall z \in(0, L) .
$$

Here, the number $c_{12}$ is constant. Using (13) and (45) and applying the CauchyBunyakovski inequality, we obtain

$$
\begin{aligned}
\left|J_{\alpha v_{0}}^{\prime}(v+\Delta v)-J_{\alpha v_{0}}^{\prime}(v)\right| \leq & \left\|\psi_{\Delta}(\cdot, z)\right\|_{L_{2}(0, l)}\|\Delta \varphi(\cdot, z)\|_{L_{2}(0, l)} \\
& +\|\Delta \psi(\cdot, z)\|_{L_{2}(0, l)}\|\varphi(\cdot, z)\|_{L_{2}(0, l)}+2 \alpha\left|\Delta v_{0}(z)\right|, \quad \forall z \in(0, L),
\end{aligned}
$$

and then

$$
\begin{aligned}
& \left\|J_{\alpha v_{0}}^{\prime}(v+\Delta v)-J_{\alpha v_{0}}^{\prime}(v)\right\|_{L_{2}(0, l)}^{2} \\
& \leq 3 \int_{0}^{L}\left\|\psi_{\Delta}(x, z)\right\|_{L_{2}(0, l)}^{2}\|\Delta \varphi(\cdot, z)\|_{L_{2}(0, l)}^{2} d z \\
& \quad+3 \int_{0}^{L}\|\Delta \psi(\cdot, z)\|_{L_{2}(0, l)}^{2}\|\varphi(\cdot, z)\|_{L_{2}(0, l)}^{2} d z+3\left\|\Delta v_{0}\right\|_{L_{2}(0, L)}^{2} .
\end{aligned}
$$

If we use estimate (8), we can write the following inequality:

$$
\left\|\psi_{\Delta}(\cdot, z)\right\|_{L_{2}(0, l)}^{2} \leq c_{13}, \quad \forall z \in[0, L]
$$

Using this inequality and estimates (13), (28), and (45), we obtain

$$
\left\|J_{\alpha v_{0}}^{\prime}(v+\Delta v)-J_{\alpha v_{0}}^{\prime}(v)\right\|_{L_{2}(0, L)} \leq c_{14}\|\Delta v\|_{H}
$$

Here, the number of $c_{14}$ is constant. Similarly, we can prove the following inequality:

$$
\left\|J_{\alpha v_{1}}^{\prime}(v+\Delta v)-J_{\alpha v_{1}}^{\prime}(v)\right\|_{L_{2}(0, L)} \leq c_{15}\|\Delta v\|_{H}
$$

If we use inequalities (48) and (49), we see that the correlations limit (36) and (37) is valid.

Now, we prove (38). To prove this using the formula $J_{\alpha \varphi_{1}}^{\prime}(v)=\operatorname{Re}(\bar{\varphi}(x, 0))+2 \alpha\left(\varphi_{1}(x)-\right.$ $\left.\tilde{\omega}_{1}(x)\right)$ in (29), we can write the following inequality:

$$
J_{\alpha v_{0}}^{\prime}(v+\Delta v)-J_{\alpha v_{0}}^{\prime}(v)=I_{m}(\Delta \bar{\varphi}(x, 0))+2 \alpha \Delta \varphi_{0}
$$

Here, $\Delta \varphi(x, z)$ is a solution of the problem (41). Estimate (45) is valid for $\forall z \in[0, L]$. Therefore, the following estimate can be written at $z=0$ :

$$
\|\varphi(\cdot, 0)\|_{L_{2}(0, l)}^{2} \leq c_{12}\left(\|\Delta v\|_{H}^{2}\right)
$$

If this inequality is used in (49), we easily can write

$$
\left\|J_{\alpha \varphi_{0}}^{\prime}(v+\Delta v)-J_{\alpha \varphi_{0}}^{\prime}(v)\right\|_{L_{2}(0, l)} \leq c_{16}\left(\|\Delta v\|_{H}\right) .
$$


Similarly, if we use (39), we obtain

$$
\left\|J_{\alpha \varphi_{1}}^{\prime}(v+\Delta v)-J_{\alpha \varphi_{1}}^{\prime}(v)\right\|_{L_{2}(0, l)} \leq c_{17}\left(\|\Delta v\|_{H}\right) .
$$

We can see that (38) and (39) are valid by inequalities (51) and (52). That is, $J_{\alpha} \in C^{1}(V)$. On the other hand, $V$ is a convex set according to the definition. So, the functional $J_{\alpha}(v)$ holds by the condition of Theorem (Goebel) in [8] at $V$. Therefore, considering Theorem 3, we obtain

$$
\left.\left\langle J_{\alpha}^{\prime}\left(v^{*}\right), v-v^{*}\right)\right\rangle_{H} \geq 0
$$

for $\forall z \in V$. Here, using (29), it is seen that the statement of Theorem 4 is valid.

\section{Competing interests}

The authors declare that they have no competing interests.

\section{Authors' contributions}

YK carried out the optimal control problem studies, participated in the sequence alignment and drafted the manuscript. EC conceived of the study and, participated in its design and coordination. All authors read and approved the final manuscript.

\section{Author details}

${ }^{1}$ Department of Mathematics, Ağrı Ibrahim Çeçen University Faculty of Science and Art, Ağrı, Turkey. ${ }^{2}$ Department of Mathematics, Atatürk University Faculty of Science, Erzurum, Turkey.

\section{Received: 1 October 2012 Accepted: 30 November 2012 Published: 28 December 2012}

\section{References}

1. İskenderov, AD, Yagubov, GY: Optimal control of non-linear quantum-mechanical systems. Autom. Remote Control 50, 1631-1641 (1989)

2. İskenderov, $A D$, Yagubov, $G Y$ : A variational method for solving the inverse problem of determining the quantumnmechanical potential. Sov. Math. Doklady (Engl. Trans.) Am. Math. Soc. 38, 637-641 (1989)

3. Yagubov, GY, Musayeva, MA: About the problem of identification for nonlinear Schrödinger equation. J. Differ. Equ. 33(12), 1691-1698 (1997)

4. Yagubov, GY: Optimal control by coefficient of quasilinear Schrödinger equation. Abstract of these doctors sciences, Kiev, p. 25 (1994)

5. Yetişkin, H, Subaşı, M: On the optimal control problem for Schrödinger equation with complex potential. Appl. Math. Comput. 216, 1896-1902 (2010)

6. Yıldiz, B, Yagubov, G: On the optimal control problem. J. Comput. Appl. Math. 88, 275-287 (1997)

7. Ladyzhenskaya, OA, Solonnikov, VA, Uralsteva, NN: Linear and Quasi-Linear Equations of Parabolic Type. Translation of Mathematical Monograps. Am. Math. Soc., Providence (1968)

8. Goebel, M: On existence of optimal control. Math. Nachr. 93, 67-73 (1979) 